\title{
Effect of Ambient Humidity on the Transmission of UV/Visible Light through Model Human Epidermis
}

\author{
Carlton Farley III ${ }^{1}$, Aschalew Kassu ${ }^{2 *}$, Sandra Sadate ${ }^{1}$, Anup Sharma ${ }^{1}$ \\ ${ }^{1}$ Department of Physics, Chemistry and Mathematics, Alabama A\&M University, Normal, AL, USA \\ ${ }^{2}$ Department of Engineering, Construction Management \& Industrial Technology, Alabama A \& M University, \\ Normal, AL, USA \\ Email: *aschalew.kassu@aamu.edu
}

Received 24 February 2016; accepted 26 March 2016; published 29 March 2016

Copyright (C) 2016 by authors and Scientific Research Publishing Inc.

This work is licensed under the Creative Commons Attribution International License (CC BY). http://creativecommons.org/licenses/by/4.0/

(c) (i) Open Access

\section{Abstract}

Transmission of light through model human epidermal samples is investigated at four different wavelengths and at varying ambient humidity. Light from light emitting diodes (LEDs) is used for transmission measurements through the samples at a UVA wavelength of $365 \mathrm{~nm}$, and visible wavelengths of $460 \mathrm{~nm}, 500 \mathrm{~nm}$, and $595 \mathrm{~nm}$. Ambient air-humidity is varied between $20 \%$ and $100 \%$. Results show that for high ambient humidity, near $100 \%$, transmission of light through the epidermis is higher than at low ambient humidity, $60 \%$ or lower. These results are explained with a simple model of epidermis as a turbid medium and the effect of adsorbed water in reducing light-scattering by refractive-index-matching. Biological implications of increased light-transmission through epidermis at high ambient humidity are discussed.

\section{Keywords}

Model Human Epidermis, Model Skin, Effect of Humidity, Transmission of Light, Optical Properties of Epidermis

\section{Introduction}

Human skin is made up of two main layers; the epidermis, and the dermis [1]. The dermis is a thick inner layer containing nerve endings and blood vessels. The epidermis is a thinner outer layer of skin that is made up of several sub-layers; including the outermost stratum corneum, stratum lucidum, stratum granulosum, stratum

\footnotetext{
${ }^{*}$ Corresponding author.
}

How to cite this paper: Farley III, C., Kassu, A., Sadate, S. and Sharma, A. (2016) Effect of Ambient Humidity on the Transmission of UV/Visible Light through Model Human Epidermis. Open Journal of Applied Sciences, 6, 153-157. 
spinosum, and the innermost stratum basale. The epidermis has several key functions important for our survival. It acts as a barrier for keeping water and nutrients in the body [2]. The epidermis is also the body's first line of defense against harmful radiation, such as UV and X-ray. The interaction of light with skin has been a topic of interest for many years, with such applications as early detection of melanoma by reflectance spectroscopy based on absorption of melanin [3], estimating blood oxygen content using absorption, understanding skin tanning and photo-ageing. While the Kubelka-Munk (K-M) models are scattering models that rely on input variables of reflectance and transmittance [4], Monte-Carlo simulations can be used in many ways to predict reflectance, transmittance, blood content, etc. [5] [6]. Several optical studies have focused on thickness of skin [7] [8], forward scattering [9], reflection spectra, and transmission. Most recently, light scattering properties of surface of human skin are theoretically studied using Maxwell's equation [10]. The effects of varying level of melanin in light reflectance of a wide range of wavelength are investigated for its applications in biomedical optics such as heart rate monitoring devices [11]. However, the impact of ambient humidity on the optics of the skin is poorly understood. In a recent work, it has been shown that mice kept at higher humidity were subject to greater damage from UV radiation than those kept at lower humidity [12]. A possible mechanism that was proposed to explain these results involves enhanced light transmission through hydrated stratum corneum. This effect has obvious clinical importance since it is known that under exposure to sun, wet skin burns more easily than dry skin [12]. One investigation involved hydration of epidermal samples by immersing in solution of $\mathrm{NaCl}$ in water. Transmission of UVC and UVB wavelengths was monitored as the samples were dried in air over several hours [7]. In another study, it was shown that ambient humidity has a large effect on the water content, and by extension, the barrier function of the epidermis [13]. However, to the best of our knowledge, no light transmission measurements for the effect of ambient humidity in isolated epidermis have been made to date for the important UVA and visible wavelengths present in the terrestrial solar spectrum.

\section{Methods and Materials}

The highly differentiated and multilayered model epidermal samples are procured commercially (Mattek, MEL-312-B). These are grown from human-derived epidermal keratinocytes and melanocytes and are known to exhibit several morphological and functional characteristics of human epidermis. For example they can be cultured to undergo melanogenesis. Model epidermal samples have been used in skin absorption studies and were shown to be suitable replacements for human skin. Before shipping, the samples used in this study were cultured for a week to develop measurable levels of melanin pigmentation. Soon after receiving, they were kept in a desiccator and dried till the time of use in our experiments. At the time of use, the model epidermis (10 mm diameter) was peeled off from the supporting base of the cell culture inserts and mounted on a ring for the ease of making light transmission measurements. It is placed in a humidity controlled chamber for several hours prior to use to allow time to absorb moisture and reach equilibrium with the ambient humidity.

We use a simple transmission technique which involves placing the sample in a humidity-controlled chamber, and monitor transmitted low power LED light (approx. $1 \mathrm{~mW}$ ) through the sample as the ambient humidity is varied slowly (Figure 1).

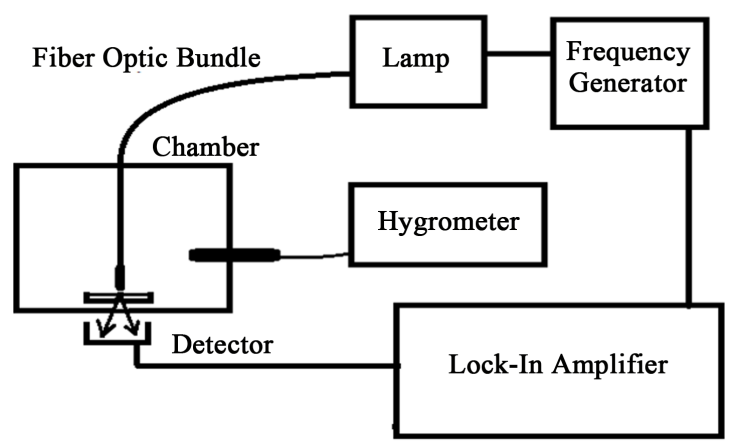

Figure 1. Schematic of the experimental set-up. LED light modulated by a frequency generator is sent through a fiber optic bundle into a humidity-controlled chamber. An epidermal sample is placed inside the chamber, where the humidity is monitored using a hygrometer. Light transmitted through the sample is measured with a photodiode and lock-in amplifier. A computer is used to analyze the data. 
Four LED light sources of different wavelengths are used; 365 nm, 460 nm, 500 nm, and 595 nm. LED light is launched through a fiber optic bundle and is modulated by a frequency generator for detection using a lock-in amplifier. Light is incident on the stratum-corneum side of the epidermal sample over an area of roughly $1 \mathrm{~mm}^{2}$. A photodiode is used for detection of transmitted light. We also used two broadband light sources (Deuterium and Halogen lamps), and performed similar measurements with them. The results yielded were almost identical, showing a greater decrease of intensity for shorter wavelengths than for longer wavelengths. However, visualization of results was much clearer using four distinct wavelengths, which are all chosen to be in the UVA-visible region (UVA and visible light from the sun penetrate Earth's atmosphere, while UVB and UVC light from the sun does not).

\section{Results and Discussion}

The curves in Figure 2 show the transmitted light intensity through the model epidermis as the humidity is gradually lowered from near $100 \%$ to $60 \%$. The near $100 \%$ humidity is produced by keeping a wet sponge inside the closed humidity chamber. At the start of the experiment, the wet sponge is removed and replaced with a small quantity of calcium chloride which dries the air. This gradually decreases the ambient humidity to $60 \%$ in a time of about 30 minutes. These experiments were carried out several times on the same sample, as well as on different samples. With the decrease of ambient humidity in a period of over an hour, significant decrease in light transmission is noted for all four wavelengths used, ranging from approximately $2.5 \%$ for $595 \mathrm{~nm}$ light to $24 \%$ for UVA wavelength (Figure 2). Most of the decrease in transmission occurs as the humidity falls from $100 \%$ to $80 \%$. A possible explanation is that adsorbed water within the turbid medium of stratum corneum acts as an index matching fluid. The refractive index of epidermis is known to be in the 1.3 to 1.5 range [14]. These values are closer to the refractive index 1.33 of water as compared to 1.0 for air. Desorption of water from the stratum corneum as the ambient humidity is lowered is thus expected to increase light scattering and reduce transmission as seen below in Figure 2.

Since presence of adsorbed humidity in epidermis increases transmission in Figure 2, we can assume that absorption of light by the epidermal humidity does not play a significant role in explaining these results. As the epidermal samples dry, the decrease of transmitted intensity $(\Delta \mathrm{I})$ for humidity change from $100 \%$ to $60 \%$ is measured from the plots in Figure 2 to be 2.5\%, 5\%, 8\% and 24\% for wavelength of $595 \mathrm{~nm}, 500 \mathrm{~nm}, 460 \mathrm{~nm}$ and $365 \mathrm{~nm}$ respectively. We believe that this increase in transmission (decrease in scattering) is due to refractive index matching between adsorbed water and the epidermal tissue and is observed in many media which are highly turbid. This is illustrated with a simple example of light transmission through a thin layer of finely crushed glass as shown in the inset of Figure 2. Wetting of this turbid medium with a drop of water results in an observable increase of light transmission due to refractive-index matching between water and glass. This is seen in the inset, Figure 2(f). The signal in Figure 2(e) appears noisier than the other measurements because of two reasons. First, we are detecting low-power $(1 \mathrm{~mW})$ diffuse lamp light transmitted through turbid media, while measuring relatively small decreases in light intensity (24\%) over a 30 minute period. Second, we believe that the sensitivity of the detectors is most likely better for visible light than for UVA light.

The decrease of transmitted intensity $(\Delta \mathrm{I})$ for humidity change from $100 \%$ to $60 \%$ is plotted logarithmically with wavelength in Figure 3. The slope of $-4.53 \pm 0.65$ for this logarithmic plot is indicative of Rayleigh scattering as the dominant light-scattering mechanism in the model epidermal samples. This conclusion supports earlier work [15] where Rayleigh scattering was found to be dominant over Mie scattering.

Significant change of UV/visible light transmission through the epidermis is seen only for high ambient humidity of over $80 \%$ (Figure 2). This can be explained from the known characteristics of adsorption isotherms for stratum corneum [16]. The moisture content of stratum corneum is a supra-linear function of ambient relative humidity. As an example, for a change of relative air-humidity from $40 \%$ to $80 \%$, the adsorbed humidity within the stratum corneum can increase by an order of magnitude. For relative air-humidity of higher than $80 \%$, the increase of adsorbed moisture in epidermis is even more rapid.

We believe this decrease of scattered and reflected light is caused by adsorbtion of water largely within the stratum corneum. By filling the voids in the uppermost layer of skin it reduces the spatial variations of refractive index and consequently the scattering of incident light. This role of water as a refractive-index matching fluid in porous and turbid medium like skin is commonly known [15] and also illustrated in the inset of Figure 2.

The results described above have obvious clinical importance. It is known that wet skin is prone to sun-burn 


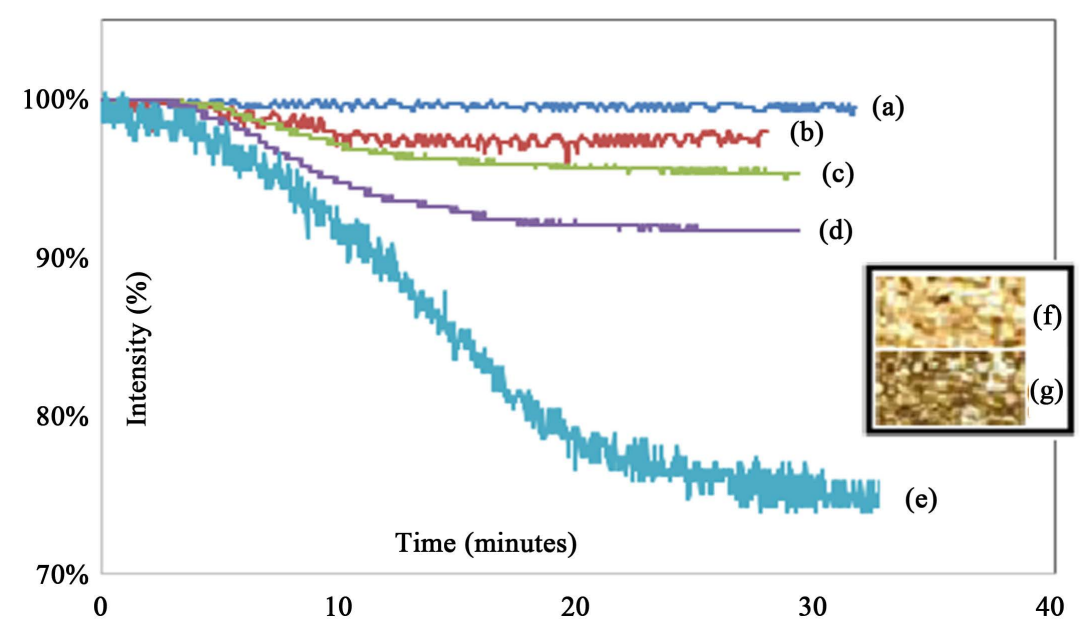

Figure 2. Intensity versus time for low power transmitted light through air-dried model-epidermis samples. To begin with, samples in the chamber are at near $100 \%$ ambient humidity. In a time of 30 minutes, the humidity decreases to around $60 \%$. Curves (b), (c), (d), (e) are for wavelengths of $595 \mathrm{~nm}$, $500 \mathrm{~nm}, 460 \mathrm{~nm}$, and $365 \mathrm{~nm}$ respectively. Curve (a) represents a baseline measurement in which no sample is present. All curves have been normalized for easy visualization and comparison. The inset is for illustrating the effect of moisture on light transmission through a turbid medium (finely crushed glass). The inset (f) and (g) shows light transmission with and without moisture, respectively.

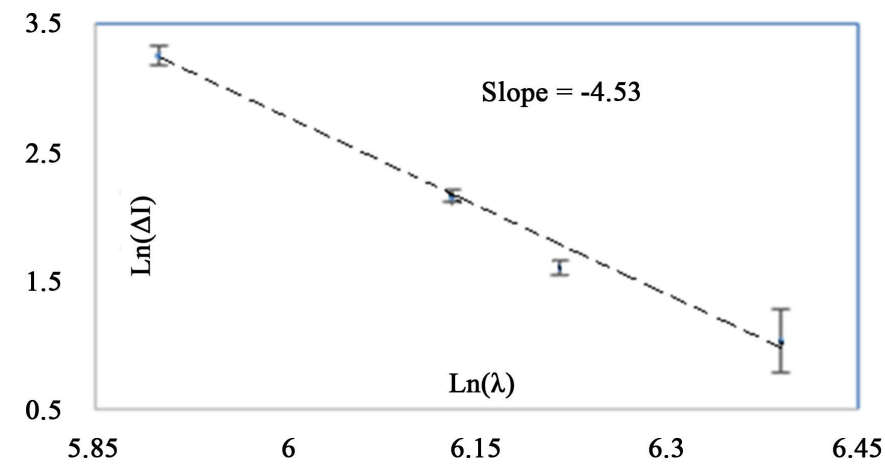

Figure 3. Logarithmic plot of $\Delta \mathrm{I}$ (Figure 2) with wavelength. A slope of $-4.53 \pm 0.65$ is indicative of Rayleigh scattering as the dominant mechanism to explain effect of ambient humidity on transmission through model human epidermis.

more than dry skin [12]. It is also known that skin can become less reflecting and more transmitting to light by prolonged bath in water or by applying a lipophilic liquid which helps in "refractive-index matching" within stratum corneum [15]. Our results show that all of the above conclusions could also happen under conditions of high humidity in ambient air. Our results could also be important for explaining the role of environmental effects on the evolution of melanism. It is known [17] that warm-blooded biological species in humid habitats show higher degree of melanin pigmentation (Gloger's rule). A possible explanation for Gloger's rule could be that additional melanin pigmentation is necessary to compensate for the effect of greater transmission of UV light through the skin under humid ambient conditions.

\section{Conclusion}

Although this work does not include UVB (280 - $315 \mathrm{~nm}$ ), we expect similar conclusions for all UV-visible wavelengths including UVB which has implications for cancer research, skin tanning, sun-burn as well as vitamin D production by skin. In conclusion, this work shows a significant effect of ambient air-humidity on the transmission of light through the epidermis and these results must be embedded in any mathematical model for explaining the optical properties of epidermis including light scattering. 


\section{Acknowledgements}

The authors acknowledge the grant from National Science Foundation, NSF-EPSCoR award number EPS1158862 for supporting this study.

\section{References}

[1] Sherwood, L. (2008) Human Physiology: From Cells to Systems. Cengage Learning, USA.

[2] Elias, P.M. and Feingold, K.R. (1999) Fitzpatrick’s Dermatology in General Medicine: Skin as an Organ of Protection. McGraw-Hill, USA.

[3] Zonios, G., Dimou, A., Bassukas, I., Galaris, D., Tsolakidis, A. and Kaxiras, E. (2008) Melanin Absorption Spectroscopy: New Method for Noninvasive Skin Investigation and Melanoma Detection. Journal of Biomedical Optics, 13, Article ID: 014017. http://dx.doi.org/10.1117/1.2844710

[4] Diffey, B. (1983) A mathematical Model for Ultraviolet Optics in Skin. Physics in Medicine and Biology, 28, $647-657$. http://dx.doi.org/10.1088/0031-9155/28/6/005

[5] Shimada, M., Yamada, Y., Itoh, M. and Yatagai, T. (2001) Melanin and Blood Concentration in a Human Skin Model Studied by Multiple Regression Analysis: Assessment by Monte Carlo Simulation. Physics in Medicine and Biology, 46, 2397-2406. http://dx.doi.org/10.1088/0031-9155/46/9/309

[6] Zeng, H., Macaulay, C., Mclean, D. and Palcic, B. (1997) Reconstruction of In-Vivo Skin Autofluorescence Spectrum from Microscopic Properties by Monte Carlo Simulation. Journal of Photochemistry and Photobiology B: Biology, 38, 234-240. http://dx.doi.org/10.1016/S1011-1344(96)00008-5

[7] Bruls, W.A.G. and Slaper, H., Van Der Leun, J.C. and Berrens, L. (1984) Transmission of Human Epidermis and Stratum Corneum as a Function of Thickness in the Ultraviolet and Visible Wavelengths. Photochemistry and Photobiology, 40, 485-494. http://dx.doi.org/10.1111/j.1751-1097.1984.tb04622.x

[8] Abdussamad, A.H. (2013) An Optics Study of Mammal Skin and its Transmission Properties. MS Thesis, University of Missouri, Columbia.

[9] Bruls, W.A.G. and Van Der Leun, J.C. (1984) Forward Scattering Properties of Human Epidermal Layers. Photochemistry and Photobiology, 40, 231-242. http://dx.doi.org/10.1111/j.1751-1097.1984.tb04581.x

[10] Barun, V.V. and Ivanov, A.P. (2013) Light Scattering by a Rough Surface of Human Skin. 1. The Luminance Factor of Reflected Light. Quantum Electronics, 43, 768-776. http://dx.doi.org/10.1070/QE2013v043n08ABEH015074

[11] Fallow, A.B., Tarumi, T. and Tanaka, H. (2013) Influence of Skin Type and Wavelength on Light Wave Reflectance. Journal of Clinical Monitoring and Computing, 27, 313-317. http://dx.doi.org/10.1007/s10877-013-9436-7

[12] Owens, D.W., Knox, J.M. and Hudson, H.T. (1975) Troll Influence of Humidity on Ultraviolet Injury. Journal of Investigative Dermatology, 64, 250-252. http://dx.doi.org/10.1111/1523-1747.ep12510673

[13] Denda, M., Sato, J., Masuda, Y., Tsuchiva, T., Kovama, J., Kuramoto, M., Elias, P.M. and Feingold, K.R. (1998) Exposure to a Dry Environment Enhances Epidermal Permeability Barrier Function. Journal of Investigative Dermatology, 111, 858-863. http://dx.doi.org/10.1046/j.1523-1747.1998.00333.x

[14] Tearney, G.J. (1995) Determination of the Refractive Index of Highly Scattering Human Tissue by Optical Coherence Tomography. Optics Letters, 21, 2258-2260. http://dx.doi.org/10.1364/OL.20.002258

[15] Saidi, I.S., Jacques, S.L. and Tittel, F.K. (1995) Mie and Rayleigh Modeling of Visible-Light Scattering in Neonatal Skin. Applied Optics, 31, 7410-7418. http://dx.doi.org/10.1364/AO.34.007410

[16] Kasting, G.B. and Barai, N.D. (2003) Equilibrium Water Sorption in Human Stratum Corneum. Journal of Pharmaceutical Sciences, 92, 1624-1631. http://dx.doi.org/10.1002/jps.10420

[17] Burtt, E.H. and Ichida, J.M. (2004) Gloger’s Rule, Feather-Degrading Bacteria, and Color Variation. The Condor, 106, 681-686. http://dx.doi.org/10.1650/7383 